\title{
ANNOTATION AND ANALYSIS OF THE TRANSCRIPTOME OF BEARING BRANCH
}

\author{
ZHU, B. ${ }^{1}-$ ZhANG, L. $^{1 *}-$ XU, X. B. ${ }^{1}-$ ZoU, B. Y. ${ }^{2}-$ GUAN, Q. M. ${ }^{2}-$ YANG, J. J. $^{2}-$ YU, Z. ${ }^{2}$ \\ ${ }^{1}$ Forestry College, Jiangxi Agricultural University, Nanchang 330045, China \\ ${ }^{2}$ Jiangxi BoJun Agriculture Holding Limited, Nancheng 344700, China \\ ${ }^{*}$ Corresponding author \\ e-mail:LLPPMM222@163.com
}

(Received $4^{\text {th }}$ Jun 2019; accepted $16^{\text {th }}$ Oct 2019)

\begin{abstract}
The analysis of the Transcriptome of different jujube, and the mining of their functional genes was performed. And the genes were annotated and analyzed, and the difference gene was screened out to provide the theoretical basis for the fresh jujube. The sequencing of the cDNA library was performed using synthesis technology and the Illumina HiSeq platform. The sequencing results of splicing and functional annotation. A total of 29402 Unigenes were annotated, while in the Nr, Swiss-Prot, GO, COG, KEGG and Pfam databases 29330, 24358, 21771, 11028, 6962 and 28940 Unigenes, respectively. At the same time, 355 new genes were annotated. The 50 functional groups in the Go database gene function annotation, 14608 metabolic processes in biological processes, accounting for 67.1\%; reference KEGG database, 110 metabolic pathways, the number of annotations, gene function ribosomes, amino acid biosynthesis. Human embryonic stem cells, carbon metabolism, and plant hormone signaling are more abundant; it is noted that COD in 11028 single-gene distribution families has general function prediction, transcription, replication, recombination and repair, and secondary metabolites in 25 genes. Biosynthesis, transport and catabolism. This study is the first time to use RNA-seq high-throughput sequencing technology to perform transcriptome sequencing and functional analysis of transcriptome results in fresh-eating jujube leaves, to explore genes for biological processes, cell components and molecular functions, and to study different functional genes, effective biosynthetic pathways and regulatory mechanisms.
\end{abstract}

Keywords: fresh jujube, Bearing branch, RNA-seq, differential gene expression, gene ontology, transcriptomics

\section{Introduction}

Ziziphus jujuba Mill is a plant belonging to the Rhamnaceae family. It's the main cultivated species in China. Cultivation and distribution all over the country. The species has recently been introduced to Asia, Europe, America, Africa and Oceania, but is generally not cultivated economically (Qu et al., 1993). Jujube are different from other fruits. Its branches can be divided into once branch of jujube, jujube stock and bearing branch. The Bearing branch is the basic unit of the result of the jujube tree. It is also known as the result branch, the exfoliative branch, the falling branch, and the two type 
branch (Tang et al., 2012). Bearing branch can be divided into lignification jujube hoists and shedding jujube cranes (Yang et al., 2007, 2014). Recently, many studies have been made on the lignification of jujube cranes. Scholars believe that lignification jujube is better in result performance. Study on the cultivation of fresh Chinese jujube in the South. Study on the performance, photosynthetic efficiency, nutrient transport and nutrient accumulation of lignification jujube hoist (Yan et al., 2013; Wang et al., 2014). The majority of jujube stock is formed by the accessory bud. In practice, callus or pruning of jujube head is easy to form jujube crane. The jujube cranes can also be lifted on the base of the jujube head and the two branches of the year. Most jujube cranes are able to blossom, and the small part of the jujube is suspended from the top of the jujube head (Shi et al., 1999; Tang et al., 2012; Wang et al., 2014). In fact, jujube crane can be divided into fruit jujube crane and non-fruiting jujube crane from its form, bear fruit Jujube cranes are divided into lignification jujube cranes and non-lignification jujube cranes. The study of jujube crane is directly related to the value of the breeding and breeding of jujube trees. It is also related to the economic and production value of jujube trees. Study the development rule of jujube crane to provide basic theory for production management (Sun et al., 1996; Chen et al., 2015).

RNA-Seq can respond to the expression of genes at the transcriptional level (Reich et al., 2018). High energy sequencing can be used to better study the expression of different genes in different jujube cranes. Thus, the molecular formation mechanism of the flower bud differentiation and results of jujube crane is described better.

\section{Materials and methods}

\section{Experimental materials}

Sample from Jiangxi Xianlv modern agricultural demonstration garden Magu jujube No. 1, Take 3 different jujube cranes: first is Lignification jujube hanging leaf (A1, A2, A3), Non lignification jujube hanging leaf (B1, B2, B3). Non lignification jujube leaves without flower bud differentiation $(\mathrm{C} 1, \mathrm{C} 2, \mathrm{C} 3)$. Lignification leaves of jujube leaves on 3 jujube trees (A1, A2, A3), B1 and C1 are the same trees, B2 and C2 are the same trees, B3 and C3 are the same trees. A total of 9 samples (3 Biological duplicates). Rapid freezing of liquid nitrogen after sampling. Put in the $-80^{\circ} \mathrm{C}$ refrigerator and spare.

\section{Construction of cDNA library and sequencing of Transcriptome}

RNA sequencing is commissioned by Hangzhou Jing Jie Biological Technology Co., Ltd. After testing the sample. Magnetic beads with Oligo (dT). Complementary pairing through A-T and use The combination of mRNA's ployA tail enriching the mRNA of eukaryotes. Then add fragmentation buffer to break mRNA into short segments. Take mRNA as a template. Synthesis of a chain cDNA with six base random primers. And then add the buffer, $d N T P s$ and DNA polymerase I. Synthesis of two chain cDNA. Then using 
AMPure XP beads to purify double stranded cDNA. Purification of double stranded cDNA for terminal repair. Adding A tail and connecting sequenced joint. Then AMPure $\mathrm{XP}$ beads is used to select the size of the fragment. Finally, the final PCR library is enriched and the final cDNA library is obtained (Qi et al., 2011; Ding et al., 2017).

\section{Statistics and comparison of sequencing}

The original data reads of the machine. Clean Data was filtered after removing the reads containing the joint, repeated and low quality of the sequencing. Sequence alignment with the specified reference genome. The obtained Mapped Data. Quality evaluation of insert fragment length test, randomness test and so on. This project uses the specified genome as a reference for sequence alignment and subsequent analysis. Using TopHat2 (Kim et al., 2013) Alignment of Clean Reads with the reference genome. Obtaining location information on the reference genome or gene. And sequence characteristic information of sequence samples. TopHat 2 is based on the comparison of software Bowtie2 (Langmead et al., 2012). Comparison of the transcriptome sequence Reads to the genome, Recognition of splice points between exons by analyzing comparison results (Splicing Junction).

\section{Discovery of new genes}

Based on the selected reference genome sequence. Using Cufflinks software to splice Mapped Reads. And compare with the original genome annotation information. Looking for the original unnoted transcriptional area. Discovery of the new transcript and new genes of the species. So as to supplement and improve the original genome annotation information. Filtering the encoded peptide chain is too short or contains only a single exon sequence (less than 50 amino acid residues). Using BLAST software to compare the new genes that are excavated with the NR, Swiss-Prot, GO, COG, KOG, Pfam, KEGG database, KEGG Orthology results of new genes were obtained by using KOBAS2.0, Using HMMER software compared with Pfam database after predicting the amino acid sequence of the new gene. Annotated information of new genes (Zhu et al., 2018).

\section{Functional annotation and analysis of the Transcriptome}

Filtering and assembly of Unigenes. Based on the selected reference genome sequence. Using Cufflinks software to splice Mapped Reads. And compare with the original genome annotation information. Looking for the original unnoted transcriptional area. Discovery of the new transcript and new genes of the species. So as to supplement and improve the original genome annotation information. Filtering the encoded peptide chain is too short or contains only a single exon sequence (less than 50 amino acid residues). Using BLAST software to compare the new genes that are excavated with the NR, Swiss-Prot, GO, COG, $K O G, P f a m, K E G G$ database. KEGG Orthology results of new genes were obtained by using KOBAS2.0. Using HMMER software compared with Pfam database after predicting 
the amino acid sequence of the new gene, Obtain annotated information of the measured genes (Liu et al., 2017; Niu et al., 2018; Zhang et al., 2018; Chen et al., 2018).

\section{Results and analysis}

\section{Sequencing data output statistics}

Repeat sequencing of 3 material samples with high energy sequencing technology. The total number of pair-end Reads of clean Data in the ReadSum of A2 is maximum. Up to 28708241. The total base number of Clean Data in BaseSum is 8612472300. The minimum of A3's ReadSum in all the samples is 21709049. The BaseSum 6512714700 of A3. The percentage of two bases of $\mathrm{G}$ and $\mathrm{C}$ in Clean DataGC is more than $44 \%$. The percentage of Clean Data with a mass greater than or equal to 30 of a base is greater than $88 \%$. The percentage of Clean Data with a mass greater than or equal to 20 of a base is greater than $94 \%$. The above analysis indicates that the quality of sequencing is good, and the data can be used for subsequent All-Unigenes database establishment, gene function annotation, classification and same-sex analysis. The output statistics of the sample data in this project are shown in Table 1.

Table 1. Sequencing data statistics table

\begin{tabular}{c|c|c|c|c|c}
\hline SampleID & ReadSum & BaseSum & GC(\%) & Q20(\%) & Q30(\%) \\
\hline A1 & $24,974,459$ & $7,492,337,700$ & $45.18 \%$ & $95.07 \%$ & $88.88 \%$ \\
A2 & $28,708,241$ & $8,612,472,300$ & $44.78 \%$ & $95.25 \%$ & $89.25 \%$ \\
A3 & $21,709,049$ & $6,512,714,700$ & $44.34 \%$ & $94.81 \%$ & $88.42 \%$ \\
B1 & $26,961,553$ & $8,088,465,900$ & $44.44 \%$ & $95.25 \%$ & $89.25 \%$ \\
B2 & $26,875,609$ & $8,062,682,700$ & $45.00 \%$ & $95.27 \%$ & $89.26 \%$ \\
B3 & $27,843,858$ & $8,353,157,400$ & $44.96 \%$ & $95.28 \%$ & $89.30 \%$ \\
C1 & $22,799,545$ & $6,839,863,500$ & $44.79 \%$ & $95.25 \%$ & $89.24 \%$ \\
C2 & $24,859,991$ & $7,457,997,300$ & $45.08 \%$ & $95.34 \%$ & $89.41 \%$ \\
C3 & $24,962,466$ & $7,488,739,800$ & $45.00 \%$ & $95.32 \%$ & $89.35 \%$ \\
\hline
\end{tabular}

\section{Ratio of transcriptional data to reference genome sequence}

This not only provides a data base for variable splicing analysis, but also enables more Reads to be compared to the reference genome, and improves the utilization of sequencing data.

Comparison efficiency refers to the percentage of Mapped Reads that accounts for Clean Reads, which is the most direct manifestation of the data utilization of the transcriptional group. The efficiency of comparison is not only affected by the quality of data sequencing, but also related to the advantages and disadvantages of the reference genome assembly, and the biological classification relationship between reference genome and sequencing samples. By comparing efficiency, it is possible to assess whether the selected reference genome assembly can meet the needs of information analysis (Table 2). 
Table 2. A statistical table of sequence alignment of the sample sequence and the selected reference genome

\begin{tabular}{c|c|c|c|c|c|c}
\hline sampleID & total_read & $\begin{array}{c}\text { reads_mapped } \\
\mathbf{( \% )}\end{array}$ & $\begin{array}{c}\text { multi_mapped } \\
\mathbf{( \% )}\end{array}$ & $\begin{array}{c}\text { uniq_mapped } \\
(\boldsymbol{\%})\end{array}$ & $\begin{array}{c}\text { reads_plus } \\
(\boldsymbol{\%})\end{array}$ & $\begin{array}{c}\text { reads_minus } \\
(\boldsymbol{\%})\end{array}$ \\
\hline \multirow{2}{*}{$\mathrm{A} 1$} & \multirow{2}{*}{49948918} & 34289534 & 486538 & 33802996 & 37956271 & 22435941 \\
& & $(68.6)$ & $(1.42)$ & $(98.58)$ & $(62.85)$ & $(37.15)$ \\
A2 & 57416482 & 39460995 & 484144 & 38976851 & 42123971 & 24220188 \\
& & $(68.73)$ & $(1.23)$ & $(98.77)$ & $(63.49)$ & $(36.51)$ \\
A3 & 43418098 & 29243769 & 362679 & 28881090 & 32184159 & 18019123 \\
& & $(67.35)$ & $(1.24)$ & $(98.76)$ & $(64.11)$ & $(35.89)$ \\
B1 & 53923106 & 36479830 & 477714 & 36002116 & 39978099 & 22423068 \\
& & $(67.65)$ & $(1.31)$ & $(98.69)$ & $(64.07)$ & $(35.93)$ \\
B2 & \multirow{2}{*}{53751218} & 36611439 & 487630 & 36123809 & 40024889 & 22814098 \\
& & $(68.11)$ & $(1.33)$ & $(98.67)$ & $(63.69)$ & $(36.31)$ \\
B3 & \multirow{2}{*}{55687716} & 38058734 & 524265 & 37534469 & 41600309 & 23922692 \\
& & $(68.34)$ & $(1.38)$ & $(98.62)$ & $(63.49)$ & $(36.51)$ \\
C1 & \multirow{2}{*}{45599090} & 31073411 & 426693 & 30646718 & 34299648 & 19678520 \\
& & $(68.14)$ & $(1.37)$ & $(98.63)$ & $(63.54)$ & $(36.46)$ \\
C2 & \multirow{2}{*}{49719982} & 33687509 & 499050 & 33188459 & 37803787 & 21683112 \\
& & $(67.75)$ & $(1.48)$ & $(98.52)$ & $(63.55)$ & $(36.45)$ \\
C3 & \multirow{2}{*}{49924932} & 34395909 & 478825 & 33917084 & 37594222 & 21969233 \\
& & $(68.90)$ & $(1.39)$ & $(98.61)$ & $(63.12)$ & $(36.88)$ \\
\hline
\end{tabular}

More than $67 \%$ samples in total percentage of Reads compared to the reference genome Reads number in Clean Reads accounted for the percentage of the number of Reads, compared to the reference genome at various locations in the Clean Reads accounted for less than $2 \%$, the number of Reads percentage compared to the reference genome location in Clean Reads only accounted for more than $98 \%$, the percentage ratio the number of Reads positive reference genome chains in Clean Reads accounted for more than $62 \%$ (including more than reads), compared to the number of Reads and percentage of negative strand genome reference in Clean Reads accounted for less than $38 \%$ (including more than reads).

\section{Discovery of new genes}

The total number of newly discovered genes obtained from corresponding database annotation information was 355, of which 196 were 300-1000bp in length and 155 in length $1000 \mathrm{bp}$. COG notes to 37, of which there are 19 300-1000bp in length, length of more than 1000bp 18; GO notes to 205, of which there are 123 300-1000bp in length, length of more than $1000 \mathrm{bp} 79$; KEGG notes to 65 , of which there are $38300-1000 \mathrm{bp}$ in length, length of more than $1000 \mathrm{bp}$ there are 27 ; KOG notes to 155 , of which there are 77 300-1000bp in length, length of more than 1000bp 77; Pfame notes to 138, of which there are 68 300-1000bp in length, length of more than 1000bp 68; Swissprot notes to 196 , of which there are $100300-1000 \mathrm{bp}$ in length a length of more than $1000 \mathrm{bp}, 95$; NR notes to 344, of which there are $188300-1000 \mathrm{bp}$ in length, length of more than $1000 \mathrm{bp}$ 152. The specific distribution of the genes for each data annotation is shown in Table 3 and Figure 1. 
Table 3. New discovery of gene functional annotation results

\begin{tabular}{c|c|c|c}
\hline Anno_Database & Annotated_Number & 300<=length $<\mathbf{1 0 0 0}$ & length $>=\mathbf{1 0 0 0}$ \\
\hline COG & 37 & 19 & 18 \\
GO & 205 & 123 & 79 \\
KEGG & 65 & 38 & 27 \\
KOG & 155 & 77 & 77 \\
Pfam & 138 & 68 & 68 \\
Swissprot & 196 & 100 & 95 \\
nr & 344 & 188 & 152 \\
All & 355 & 196 & 155 \\
\hline
\end{tabular}

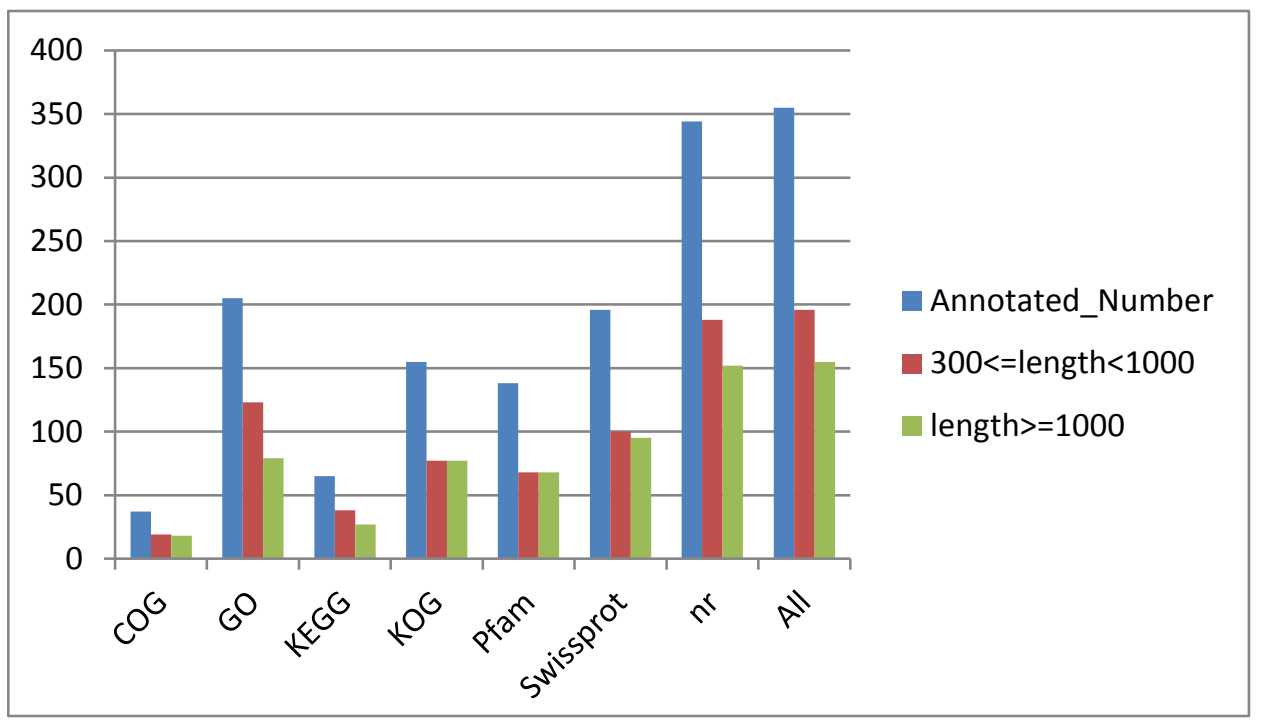

Figure 1. New gene annotation distribution

\section{Annotation and functional classification of genes}

The total number of genes in the annotation was 29402, of which 12539 were $300-1000 \mathrm{bp}$ in length and 16307 in length greater than that of $1000 \mathrm{bp}$. COG notes to 11028 , of which there are 3757 300-1000bp in length, length of more than 1000bp 7172; GO notes to 21771 , of which there are $8900300-1000 \mathrm{bp}$ in length, length of more than 1000bp 12413; KEGG notes to 6962, of which there are 2683 300-1000bp in length, length of more than $1000 \mathrm{bp}$ there are 4154 ; KOG notes to 16821 , of which there are 6294 $300-1000 \mathrm{bp}$ in length, length of more than 1000bp 10278; Pfame notes to 28940, of which there are 12273 300-1000bp in length, length of more than 1000bp 16115; Swissprot note to 24358 , which is $300-1000 \mathrm{bp}$ in length 9435 , length of more than $1000 \mathrm{bp} 14589$; NR notes to 29330, of which there are $12491300-1000 \mathrm{bp}$ in length, length of more than 1000 bp 16286.

The number of functional genes that are ultimately annotated by each database is shown in Table 4. 
Table 4. Statistical analysis of functional annotation of gene

\begin{tabular}{c|c|c|c}
\hline Anno_Database & Annotated_Number & 300<=length $<\mathbf{1 0 0 0}$ & length $>=\mathbf{1 0 0 0}$ \\
\hline COG & 11028 & 3757 & 7172 \\
GO & 21771 & 8900 & 12413 \\
KEGG & 6962 & 2683 & 4154 \\
KOG & 16821 & 6294 & 10278 \\
Swissprot & 24358 & 9435 & 14589 \\
Pfam & 28940 & 12273 & 16115 \\
nr & 29330 & 12491 & 16286 \\
All & 29402 & 12539 & 16307 \\
\hline
\end{tabular}

\section{Gene GO classification}

GO database is a structured standard biological annotation system built by GO Organization (Gene Ontology Consortium) in 2000, aiming at establishing standard vocabulary system of genes and their product knowledge, which is suitable for all kinds of species. The GO annotation system is a directed acyclic graph, which contains three main branches, namely Biological Process, Molecular Function and Cellular Component. In this study, the Unigene obtained from three samples of fresh jujube was compared in the GO functional database. A total of 21771 annotations were selected in 50 functional groups of GO biology. The metabolic processes involved in biological processes (14608, $67.1 \%$ ), cell processes $(12146,55.79 \%)$, and single biological processes (10464, $48.06 \%)$. The main components related to annotation of cell components were cells (9818, 45.09\%), membrane (8930, 41.02\%), cell components $(9760,44.83 \%)$, and membrane $(6609,30.36 \%)$. The main parts of the molecular functional annotations are catalytic activity $(11882,54.57 \%)$, and binding $(9688,44.5 \%)$. This result also shows the main distribution of the annotation function. See Table 5.

\section{Classification of KEGG metabolic pathways}

To KEGG database as a reference, there are 6962 notes to the KEGG database, can be classified into 110 groups of gene transcription in the metabolic pathways (Table 6), which notes to the biosynthesis of ribosomes, amino acid biosynthesis, carbon metabolism, plant hormone signaling, plant pathogen interaction, splicing, RNA transport, and starch sucrose metabolism, endoplasmic reticulum protein processing, phenylpropanoid is relatively high. Glucosinolate biosynthesis and degradation of aromatic compounds, sulfur relay systems, non-homologous termination, glycosphingolipid biosynthesis - ganglion series, beet red pigment biosynthesis, lipoic acid metabolism, other types of O-glycan biosynthesis and Vancomycin resistance, caffeine metabolic pathways is to compare notes low. 
Table 5. GO annotation classification of Unigene in jujube leaf transcriptional group

\begin{tabular}{|c|c|c|}
\hline \#GO_classify1 & GO_classify2 & Trans gene \\
\hline cellular component & extracellular region & 594 \\
\hline cellular component & cell & 9818 \\
\hline cellular component & nucleoid & 17 \\
\hline cellular component & membrane & 8930 \\
\hline cellular component & virion & 81 \\
\hline cellular component & cell junction & 431 \\
\hline cellular component & extracellular matrix & 11 \\
\hline cellular component & membrane-enclosed lumen & 413 \\
\hline cellular component & macromolecular complex & 2218 \\
\hline cellular component & organelle & 6339 \\
\hline cellular component & extracellular region part & 48 \\
\hline cellular component & organelle part & 2962 \\
\hline cellular component & virion part & 81 \\
\hline cellular component & membrane part & 6609 \\
\hline cellular component & cell part & 9760 \\
\hline molecular function & protein binding transcription factor activity & 108 \\
\hline molecular function & nucleic acid binding transcription factor activity & 502 \\
\hline molecular function & catalytic activity & 11882 \\
\hline molecular function & receptor activity & 153 \\
\hline molecular function & guanyl-nucleotide exchange factor activity & 30 \\
\hline molecular function & structural molecule activity & 512 \\
\hline molecular function & transporter activity & 1435 \\
\hline molecular function & binding & 9688 \\
\hline molecular function & electron carrier activity & 154 \\
\hline molecular function & antioxidant activity & 207 \\
\hline molecular function & channel regulator activity & 1 \\
\hline molecular function & metallochaperone activity & 4 \\
\hline molecular function & enzyme regulator activity & 228 \\
\hline molecular function & protein tag & 6 \\
\hline molecular function & translation regulator activity & 1 \\
\hline molecular function & nutrient reservoir activity & 44 \\
\hline molecular function & molecular transducer activity & 303 \\
\hline biological process & reproduction & 337 \\
\hline biological process & cell killing & 3 \\
\hline biological process & immune system process & 234 \\
\hline biological process & metabolic process & 14608 \\
\hline biological process & cellular process & 12146 \\
\hline biological process & reproductive process & 827 \\
\hline biological process & biological adhesion & 49 \\
\hline biological process & signaling & 1087 \\
\hline biological process & multicellular organismal process & 1343 \\
\hline biological process & developmental process & 1785 \\
\hline biological process & growth & 281 \\
\hline biological process & locomotion & 9 \\
\hline biological process & single-organism process & 10464 \\
\hline biological process & rhythmic process & 26 \\
\hline biological process & response to stimulus & 3905 \\
\hline biological process & localization & 3404 \\
\hline biological process & multi-organism process & 525 \\
\hline biological process & biological regulation & 4495 \\
\hline biological process & cellular component organization or biogenesis & 2970 \\
\hline
\end{tabular}


Table 6. KEGG metabolic pathway annotation table

\begin{tabular}{|c|c|c|}
\hline \#pathway & pathway_id & Gene_number \\
\hline Glycolysis / Gluconeogenesis & ko00010 & 102 \\
\hline Citrate cycle (TCA cycle) & ko00020 & 39 \\
\hline Pentose phosphate pathway & ko00030 & 45 \\
\hline Pentose and glucuronate interconversions & ko00040 & 66 \\
\hline Fructose and mannose metabolism & ko00051 & 54 \\
\hline Galactose metabolism & ko00052 & 57 \\
\hline Ascorbate and aldarate metabolism & ko00053 & 40 \\
\hline Fatty acid biosynthesis & ko00061 & 44 \\
\hline Fatty acid elongation & ko00062 & 35 \\
\hline Fatty acid degradation & ko00071 & 38 \\
\hline Synthesis and degradation of ketone bodies & ko00072 & 8 \\
\hline Cutin, suberine and wax biosynthesis & ko00073 & 21 \\
\hline Steroid biosynthesis & ko00100 & 19 \\
\hline Ubiquinone and other terpenoid-quinone biosynthesis & ko00130 & 18 \\
\hline Oxidative phosphorylation & ko00190 & 89 \\
\hline Photosynthesis & ko00195 & 36 \\
\hline Photosynthesis - antenna proteins & ko00196 & 12 \\
\hline Purine metabolism & ko00230 & 128 \\
\hline Caffeine metabolism & ko00232 & 1 \\
\hline Pyrimidine metabolism & ko00240 & 101 \\
\hline Alanine, aspartate and glutamate metabolism & ko00250 & 44 \\
\hline Glycine, serine and threonine metabolism & ko00260 & 60 \\
\hline Cysteine and methionine metabolism & ko00270 & 65 \\
\hline Valine, leucine and isoleucine degradation & ko00280 & 59 \\
\hline Valine, leucine and isoleucine biosynthesis & ko00290 & 25 \\
\hline Lysine biosynthesis & ko00300 & 12 \\
\hline Lysine degradation & ko00310 & 20 \\
\hline Arginine and proline metabolism & ko00330 & 72 \\
\hline Histidine metabolism & ko00340 & 16 \\
\hline Tyrosine metabolism & ko00350 & 42 \\
\hline Phenylalanine metabolism & ko00360 & 92 \\
\hline Tryptophan metabolism & ko00380 & 27 \\
\hline Phenylalanine, tyrosine and tryptophan biosynthesis & ko00400 & 38 \\
\hline beta-Alanine metabolism & ko00410 & 46 \\
\hline Taurine and hypotaurine metabolism & ko00430 & 9 \\
\hline Selenocompound metabolism & ko00450 & 25 \\
\hline Cyanoamino acid metabolism & ko00460 & 85 \\
\hline Glutathione metabolism & ko00480 & 94 \\
\hline Starch and sucrose metabolism & ko00500 & 166 \\
\hline N-Glycan biosynthesis & ko00510 & 39 \\
\hline Other glycan degradation & ko00511 & 16 \\
\hline Other types of O-glycan biosynthesis & ko00514 & 2 \\
\hline Amino sugar and nucleotide sugar metabolism & ko00520 & 106 \\
\hline Glycosaminoglycan degradation & ko00531 & 17 \\
\hline Glycerolipid metabolism & ko00561 & 47 \\
\hline Inositol phosphate metabolism & ko00562 & 58 \\
\hline Glycosylphosphatidylinositol(GPI)-anchor biosynthesis & ko00563 & 19 \\
\hline Glycerophospholipid metabolism & ko00564 & 74 \\
\hline Ether lipid metabolism & ko00565 & 28 \\
\hline Arachidonic acid metabolism & ko00590 & 10 \\
\hline Linoleic acid metabolism & ko00591 & 11 \\
\hline alpha-Linolenic acid metabolism & ko00592 & 37 \\
\hline Sphingolipid metabolism & ko00600 & 20 \\
\hline Glycosphingolipid biosynthesis - globo series & ko00603 & 14 \\
\hline Glycosphingolipid biosynthesis - ganglio series & ko00604 & 5 \\
\hline
\end{tabular}




\begin{tabular}{|c|c|c|}
\hline \#pathway & pathway_id & Gene_number \\
\hline Pyruvate metabolism & ko00620 & 78 \\
\hline Glyoxylate and dicarboxylate metabolism & ko00630 & 51 \\
\hline Propanoate metabolism & ko00640 & 26 \\
\hline Butanoate metabolism & ko00650 & 20 \\
\hline C5-Branched dibasic acid metabolism & ko00660 & 11 \\
\hline One carbon pool by folate & ko00670 & 16 \\
\hline Carbon fixation in photosynthetic organisms & ko00710 & 52 \\
\hline Thiamine metabolism & ko00730 & 9 \\
\hline Riboflavin metabolism & ko00740 & 9 \\
\hline Vitamin B6 metabolism & ko00750 & 9 \\
\hline Nicotinate and nicotinamide metabolism & ko00760 & 14 \\
\hline Pantothenate and CoA biosynthesis & ko00770 & 30 \\
\hline Biotin metabolism & ko00780 & 17 \\
\hline Lipoic acid metabolism & ko00785 & 3 \\
\hline Folate biosynthesis & ko00790 & 11 \\
\hline Porphyrin and chlorophyll metabolism & ko00860 & 45 \\
\hline Terpenoid backbone biosynthesis & ko00900 & 46 \\
\hline Monoterpenoid biosynthesis & ko00902 & 11 \\
\hline Limonene and pinene degradation & ko00903 & 8 \\
\hline Diterpenoid biosynthesis & ko00904 & 21 \\
\hline Brassinosteroid biosynthesis & ko00905 & 9 \\
\hline Carotenoid biosynthesis & ko00906 & 33 \\
\hline Zeatin biosynthesis & ko00908 & 28 \\
\hline Sesquiterpenoid and triterpenoid biosynthesis & ko00909 & 24 \\
\hline Nitrogen metabolism & ko00910 & 29 \\
\hline Sulfur metabolism & ko00920 & 35 \\
\hline Phenylpropanoid biosynthesis & ko00940 & 143 \\
\hline Flavonoid biosynthesis & ko00941 & 23 \\
\hline Anthocyanin biosynthesis & ko00942 & 13 \\
\hline Flavone and flavonol biosynthesis & ko00944 & 9 \\
\hline Stilbenoid, diarylheptanoid and gingerol biosynthesis & ko00945 & 13 \\
\hline Isoquinoline alkaloid biosynthesis & ko00950 & 23 \\
\hline Tropane, piperidine and pyridine alkaloid biosynthesis & ko00960 & 23 \\
\hline Betalain biosynthesis & ko00965 & 5 \\
\hline Glucosinolate biosynthesis & ko00966 & 8 \\
\hline Aminoacyl-tRNA biosynthesis & ko00970 & 54 \\
\hline Biosynthesis of unsaturated fatty acids & ko01040 & 33 \\
\hline Carbon metabolism & ko01200 & 199 \\
\hline 2-Oxocarboxylic acid metabolism & ko01210 & 55 \\
\hline Fatty acid metabolism & ko01212 & 68 \\
\hline Degradation of aromatic compounds & ko01220 & 8 \\
\hline Biosynthesis of amino acids & ko01230 & 206 \\
\hline Vancomycin resistance & ko01502 & 2 \\
\hline $\mathrm{ABC}$ transporters & ko02010 & 15 \\
\hline Ribosome biogenesis in eukaryotes & ko03008 & 83 \\
\hline Ribosome & ko03010 & 230 \\
\hline RNA transport & ko03013 & 167 \\
\hline mRNA surveillance pathway & ko03015 & 109 \\
\hline RNA degradation & ko03018 & 103 \\
\hline RNA polymerase & ko03020 & 41 \\
\hline Basal transcription factors & ko03022 & 40 \\
\hline DNA replication & ko03030 & 45 \\
\hline Spliceosome & ko03040 & 171 \\
\hline Proteasome & ko03050 & 45 \\
\hline Protein export & ko03060 & 41 \\
\hline Base excision repair & ko03410 & 43 \\
\hline Nucleotide excision repair & ko03420 & 49 \\
\hline
\end{tabular}




\begin{tabular}{|c|c|c|}
\hline \#pathway & pathway_id & Gene_number \\
\hline Mismatch repair & ko03430 & 33 \\
\hline Homologous recombination & ko03440 & 49 \\
\hline Non-homologous end-joining & ko03450 & 7 \\
\hline Phosphatidylinositol signaling system & ko04070 & 56 \\
\hline Plant hormone signal transduction & ko04075 & 188 \\
\hline Ubiquitin mediated proteolysis & ko04120 & 96 \\
\hline Sulfur relay system & ko04122 & 8 \\
\hline SNARE interactions in vesicular transport & ko04130 & 27 \\
\hline Regulation of autophagy & ko04140 & 21 \\
\hline Protein processing in endoplasmic reticulum & ko04141 & 157 \\
\hline Endocytosis & ko04144 & 135 \\
\hline Phagosome & ko04145 & 67 \\
\hline Peroxisome & ko04146 & 74 \\
\hline Plant-pathogen interaction & ko04626 & 180 \\
\hline Circadian rhythm - plant & ko04712 & 46 \\
\hline
\end{tabular}

\section{COG functional classification}

In this study, 11028 Unigenes, annotated into COG, were distributed in 25 gene families (Table 7).

Table 7. The function table annotated in the COG Library

\begin{tabular}{c|c|c}
\hline ID & Class_Name & Numbers \\
\hline R & General function prediction only & 3500 \\
K & Transcription & 1767 \\
L & Replication, recombination and repair & 1749 \\
T & Signal transduction mechanisms & 1669 \\
O & Posttranslational modification, protein turnover, chaperones & 960 \\
G & Carbohydrate transport and metabolism & 821 \\
J & Translation, ribosomal structure and biogenesis & 797 \\
Q & Secondary metabolites biosynthesis, transport and catabolism & 749 \\
E & Amino acid transport and metabolism & 740 \\
S & Function unknown & 615 \\
C & Energy production and conversion & 562 \\
P & Inorganic ion transport and metabolism & 530 \\
I & Lipid transport and metabolism & 457 \\
M & Cell wall/membrane/envelope biogenesis & 417 \\
D & Cell cycle control, cell division, chromosome partitioning & 310 \\
V & Defense mechanisms & 244 \\
H & Coenzyme transport and metabolism & 235 \\
A & RNA processing and modification & 185 \\
Z & Cytoskeleton & 182 \\
U & Intracellular trafficking, secretion, and vesicular transport & 180 \\
F & Nucleotide transport and metabolism & 147 \\
B & Chromatin structure and dynamics & 87 \\
N & Cell motility & 25 \\
Y & Nuclear structure & 1 \\
W & Extracellular structures & 0 \\
\hline
\end{tabular}

Such as general function prediction, transcription, replication, recombination and repair, signal transduction, signal transduction, signal transduction, post-translational modification, protein turnover, mate, sugar transport and metabolism, translation, 
ribosome structure and biosynthesis of secondary metabolites biosynthesis, transport and catabolism and other functions (Allen et al., 2018; Astaneh et al., 2018; Li et al., 2018; Nisavic et al., 2018; Wang et al., 2018; Wu et al., 2018).

\section{Conclusion}

The sequence of cDNA library using Using Illumina HiSeq platform, the sequencing results were linked and functional annotations were analyzed to reveal the overall functional expression pattern of transcripts from different leaves of jujube. In recent years, there have been many studies based on high-energy sequencing, including Daphne odora Thunb (Yang et al., 2017), Michelia macclurei Dandy (Li et al., 2017), Rhododendron calvescens Balf.f. (Li et al., 2017), Carthamus tinctorius L. (Dong et al., 2017), Penthorum chinense Pursh (Yuan et al., 2017) and other plants. Transcriptome genes are classified into 110 metabolic pathways, including ribosome, amino acid biosynthesis, carbon metabolism, plant hormone signal transduction, plant pathogen interaction, splicer, RNA transport, starch and sucrose metabolism, endoplasmic reticulum protein processing, phenylpropanoid biosynthesis and so on. This study is the first time to use RNA-seq high-throughput sequencing technology to perform transcriptome sequencing and functional analysis of transcriptome results in fresh-eating jujube leaves, to explore genes for biological processes, cell components and molecular functions, and to study different functional genes, effective biosynthetic pathways and regulatory mechanisms.

\section{Analysis of lignin-related genes in lignified jujube suspension}

The results showed that the expression of lignin-related genes was more active in comparison with non-lignified jujube suspension, all of which were up-regulated genes. There were 11 up-regulated genes: gene 29690, gene 4518, gene 30089, gene 1272, gene 21988, gene 16475, gene 16475, gene 14268, gene 14269, gene 10082 and gene 29495.

The main functional genes in gene 29690 family are monooxygenase activity and lignin metabolism. In gene 14269 gene family, it includes the functions to be expressed in almost other gene families. Meristem growth regulation, chitin decomposition, glycolysis, cytokinin response, abscisic acid response, lignin biosynthesis, polar transport of auxin, cell tip growth.

The up-regulation of gene expression related to Lignin Metabolism in lignified jujube suspension also confirms that the cause of lignified jujube suspension is related to the synergy of gene expression mentioned above. Jiang et al. (2018) found that 16 lignin genes, including $2 P A L, 5 C C R, 34 C L, 2 C A D H 2$ and $4 L A C$, were up-regulated with shoot development, suggesting that they may be related to developmental lignin accumulation. Lin et al. (2018) studied the characteristics and rules of Lignin Metabolism in okra fruits and found that the significant increase of lignin and cellulose content was 
related to phenylalanine ammonia lyase $(P A L)$, cinnamyl alcohol 4-hydroxylase $(C 4 H)$, 4 coumarin acyl-CoA ligase, protopectin and flavonoid enzymes.

$P R X 52$ is a major lignin and secondary cell wall biosynthetic gene, especially in xylem vessel. The mutant of Arabidopsis thaliana showed a 70-80\% decrease in lignin content, which was syringyl lignin (Fernandez-Perez et al., 2015). Previous studies have investigated its possible role in ABA-mediated plant defense against bacterial and fungal responses (Mohr et al., 2007). TCP3 and MYB12 were found to be involved in the regulation of PRX52 (Arro et al., 2017) in grape tendril transcriptome studies. During lignification, $P R X 52$ interacts with TCP3 more extensively than it responds to adversity. $P R X 52$ also shares the phenylpropane metabolic pathway in lignin biosynthesis with MYB12 (response switch gene for flavonol metabolism) (Czemmel et al., 2012). Phenylalanine aminolytic enzyme $(P A L)$ is a rate-limiting enzyme in the metabolic pathway of lignin phenylpropane, which catalyzes the conversion of phenylalanine to cinnamic acid and coenzyme lipids, and is one of the most important enzymes in lignin synthesis (Matus et al., 2008). In this experiment, homologous genes were expressed in the transcripts of lignified jujube suspension.

\section{Differential gene expression analysis of lignified jujube suspension related to photosynthesis}

The results showed that the genes annotated in GO were up-regulated in gene 4228, gene 10305 and gene 42934, down-regulated in gene 6204, gene 29074, gene 24178, gene 15775, gene 2674, gene 4496 and gene 296156. In the annotation of metabolic pathway in KEGG database, three genes participated in photosynthesis expression, of which gene 4496, gene 24178 were down-regulated and gene 4293 was up-regulated.

In the gene 4496 gene family, there are reactive oxygen species, photosynthesis and redox processes involved in biological processes. Molecular function is involved in iron oxidase activity and iron binding. Chloroplasts are involved in cell components. In gene 24178 expression, serine cysteine biosynthesis, photosynthetic adaptation, regulation of hydrogen peroxide metabolism, regulation of superoxide metabolism, photosynthesis and so on. The molecular functions include transferase activity, pyridoxal phosphate binding activity and cysteine synthetase activity. The thylakoid cavity is involved in the upregulation of cell composition. Gene 4293 family genes are up-regulated, including sulfur amino acid metabolism, glycine metabolism, vitamin metabolism, cell amino acid biosynthesis, aromatic amino acid metabolism, lipid metabolism, coenzyme biosynthesis, cold reaction, biological stimulation detection, blue light response. High light intensity response, red light response, far red light response, PSII related light capture complex II catabolism process, regulation of hydrogen peroxide metabolism process, chlorophyll biosynthesis process, starch biosynthesis process. Only the chloroplast envelope is involved in the expression.

Liu (2008) studied the temporal and spatial expression of $c a b-P h E 3, c a b-P h E 1, c a b-$ PhE5, $c a b-P h E 8, c a b-P h E 10$ and $c a b-P h E 11$ genes in Phyllostachys heterocycla (Carr.) 
Mitford cv. Pubescens under photosynthesis (Zhang et al., 2009). Through proteomics, bioinformatics and VIGS analysis, it was found that TYLCCNV/TYLCCNB invasion was involved in stress and defense, energy production, photosynthesis, protein homeostasis, metabolism, cell structure, signal transduction, transcription, transport and cell growth/division. Effective methylation of $\mathrm{N}$-methyl was promoted through $\mathrm{N}$-adenosineL-methionine cycle II pathway (Zhang, 2014). Molecular mechanism of carbon flux regulation is fixed carbon expression in photosynthesis in different wild-type algae and Arabidopsis thaliana. Arabidopsis thaliana is used as a model to study the transformation of genetic regulation from vegetative growth to reproductive growth (Khan et al., 2014; Carrieri et al., 2018). The study also proved that both lignified and non-lignified jujube suspension had gene expression, and more up-regulated genes were expressed in lignified jujube suspension.

\section{Summary}

By comparing lignified jujube suspension with non-lignified jujube suspension, using GO and KEGG database annotations, the differential functional genes were analyzed from three database systems.

For lignified jujube suspension analysis, 11 genes were up-regulated with lignin gene expression, and there was no up-regulated with non-lignified jujube suspension. The results showed that lignified jujube suspension was related to lignin synthesis. There are 42 genes involved in lignin biosynthesis. They have synergistic effects on Chitinase activity, polysaccharide binding, cell wall tissue, root hair elongation, regulation of carbohydrate biosynthesis, cellulose biosynthesis and meristem growth. The formation of lignified jujube suspension is related to the co-regulation of photosynthesis and endogenous hormones.

\section{REFERENCES}

[1] Allen, D. T., Carrier, D. J., Gong, J. L., Hwang, B. J., Licence, P., Moores, A., Pradeep, T., Sels, B., Subramaniam, B., Tam, M. K. C., Zhang, L., Williams, R. M. (2018): Advancing the Use of Sustainability Metrics in ACS Sustainable Chemistry \& Engineering. - ACS Publications 6(1): 1.

[2] Arro, J., Cuenca, J., Yang, Y. Z., Liang, Z. C., Cousins, P., Zhong, G. Y. (2017): A transcriptome analysis of two grapevine populations segregating for tendril phyllotaxy. Horticulture Research 4: 17032.

[3] Astaneh, R. K., Bolandnazar, S., Nahandi, F. Z., Oustan, S. (2018): The Effects of Selenium On some Physiological Traits and K, Na Concentration of Garlic (Allium Sativum L.) Under Nacl Stress. - Information Processing in Agriculture 5(1): 156-161.

[4] Carrieri, D., Lombardi, T., Paddock, T., Cano, M., Goodney, G. A., Nag, A., Old, W., Maness, P.-C., Seibert, M., Ghirardi, Yu, J. P. (2017): Transcriptome and proteome 
analysis of nitrogen starvation responses in Synechocystis $6803 \Delta \mathrm{glgC}$, a mutant incapable of glycogen storage. - Algal Research 21: 64-75.

[5] Chen, Z. L., Liu, S. P., Liu, C. H. (2015): Discussion on growth and development of jujube and jujube crane. - Chinese agronomy bulletin 31(28): 104-111.

[6] Chen, H., Wu, Y. G., Yang, Y. Z. (2018): The data assembly of the young leaves and mature leaves of patchouli and the gene function annotation. - Molecular Plant Breeding 16(7): 2139-2154.

[7] Czemmel, S., Heppel, S. C., Bogs, J. (2012): R2R3 MYB transcription factors: key regulators of the flavonoid biosynthetic pathway in grapevine. - Protoplasma 249: 109118.

[8] Ding, Y. H. (2017): High-throughput sequencing of small RNA and functional verification of microRNA in cotton male sterility induced by high temperature stress. - Huazhong Agricultural University.

[9] Dong, Y. Y., Liu, X. M., Yao, N., Li, H. Y. (2017): Transcription factor analysis based on transcriptome data of different tissues of Safflower. - Chinese herbal medicine 48(21): 4515-4522.

[10] Fernandez-Perez, F., Pomar, F., Pedreno, M. A., Novo-Uzal, E. (2015): The suppression of AtPrx 52 affects fibers but not xylem lignification in Arabidopsis by altering the proportion of syringyl units. - Physiol Plant 154: 395-406.

[11] Jiang, H. G., Wang, S. G., Liu, Y. (2018): Screening and expression analysis of lignin genes in shoots of Cizhu bamboo based on transcriptome sequencing. - Plant studies 38(03): 415421.

[12] Khan, M. R., Ai, X. Y., Zhang, J. Z. (2014): Genetic regulation of flowering time in annual and perennial plants. - Wiley Interdiscip. Rev. 5: 347-359.

[13] Kim, D., Pertea, G., Trapnell, C., Pimentel, H., Kelley, R., Salzberg, S. L. (2013): TopHat2: accurate alignment of transcriptomes in the presence of insertions, deletions and gene fusions. - Genome Biology 14(4): R36.

[14] Langmead, B., Salzberg, S. L. (2012): Fast gapped-read alignment with bowtie. - Nature Methods 9(4): 357-359.

[15] Li, Q. Y., Zhong, C. L., Jiang, Q. B. (2017): Molecular Plant Breeding 15(11): 4396-4404.

[16] Li, T. Q., Liu, X. F., Wanyou, M. (2017): Transcriptome analysis of wild plant Rhododendron longstalk based on high-throughput sequencing. - Plant studies 37(06): 825-834.

[17] Li, Z., Han, C. G. (2018): Economics of Biomass Gasification: A Review of the Current Status. - Energy Sources Part B Economics Planning \& Policy 13(2): 137-140.

[18] Lin, X., Pan, X., Kang, X. (2018): Effects of picking time on Lignin Metabolism and related enzyme activities of Chuanqiu okra. - Zhejiang Agricultural Journal 09: 1519-1525.

[19] Liu, Y. L. (2008): Isolation and expression of light-harvesting pigment binding protein gene in Phyllostachys pubescens photosystem. - Chinese Academy of Forestry Sciences.

[20] Liu, J. T., Zhu, H. S., Wen, Q. F. (2017): Separation and browning of WRKY transcription factor gene of silk gourd. - Journal of Agricultural Biotechnology 25(12): 1950-1960. 
[21] Matus, J. T., Aquea, F., Arce-Johnson, P. (2008): Analysis of the grape MYB R2R3 subfamily reveals expanded wine quality-related clades and conserved gene structure organization across Vitis and Arabidopsis genomes. - BMC Plant Biol. 8: 83.

[22] Mohr, P. G., Cahill, D. M. (2007): Suppression by ABA of salicylic acid and lignin accumulation and the expression of multiple genes, in Arabidopsis infected with Pseudomonas syringae PV. Tomato. - Funct Integr Genomics 7: 181-191.

[23] Nisavic, M., Stoiljkovic, M., Crnolatac, I., Milosevic, M., Rilak, A., Masnikosa, R. (2018): Highly Water-Soluble Ruthenium(Ii) Terpyridine Coordination Compounds Form Stable Adducts with Blood-Borne Metal Transporting Proteins. - Arabian Journal of Chemistry 11(3): 291-304.

[24] Niu, J. Q., Su, J. M., Wang, X. M. (2018): Data analysis of Dendrobium nobile leaves transcriptome based on high throughput sequencing. - Molecular Plant Breeding 16(03): 747-756.

[25] Qi, Y. X., Liu, Y. B., Rong, W. H. (2011): rRNA-Seq and its applications:a new technology for transcriptomics. - Yichuan(Hereditas) 33(11): 1191-1202.

[26] Qu, Z. Z., Wang, Y. H. (1993): Fruit tree of China. Jujube volume. - China Forestry Publishing House.

[27] Reich, I., Ijaz, U. Z., Gormally, M., Smith, C. J. (2018): 16s rRNA sequencing reveals likely beneficial core microbes within faecal samples of the eu protected slug geomalacus maculosus. - Scientific Reports 8(1): 10402.

[28] Sun, Q. H., Cheng, Z. Y., Qi, H. R. (1996): Observation and report on the growth and fruiting habit of Jinzao jujube crane. - Shaanxi forestry science and technology 3: 10-11.

[29] Tang, Z. J., He, M., Gao, J. P. (2012): Influence of pinching and sprouting on the formation of lignification jujube crane. - North horticulture 22: 26-28.

[30] Wang, S., Yan, C. (2014): Comparison of accumulation capacity of Photosynthate from 2 types of jujube crane in southern China. - Forestry Science 50(06): 90-97.

[31] Wang, S., Yan, C., Deng, B. L. (2014): Comparison of nutrient transport and accumulation ability of two types of jujube crane in southern China. - Journal of Central South University of Forestry and Technology 34(12): 35-39.

[32] Wang, M., Zhang, D. Q., Su, J., Dong, J. W., Tan, S. K. (2018): Assessing Hydrological Effects and Performance of Low Impact Development Practices Based On Future Scenarios Modeling. - Journal of Cleaner Production 179: 12-23.

[33] Wu, X., Song, M., Qiu, P., Li, F., Wang, M., Zheng, J., Wang, Q., Xu, F., Xiao, H. (2018): A Metabolite of Nobiletin, 4 '-Demethylnobiletin and Atorvastatin Synergistically Inhibits Human Colon Cancer Cell Growth by Inducing G0/G1 Cell Cycle Arrest and Apoptosis. Food \& Function 9(1): 87-95.

[34] Yan, C., Wang, S., Shao, F. X. (2013): Comparison of photosynthetic efficiency between leaves of lignification and non lignification of jujube leaves in southern jujube. - Economic Forest Research 31(02): 113-117.

[35] Yang, Y. R., Zhao, J., Liu, M. J. (2007): Research progress of jujube crane. - Acta agronomica Sinica S2: 53-57. 
[36] Yang, L., Zhang, W., Xu, Y. T. (2014): Effects of dormant pruning on Germination of ash dates, Jun jujube and date jujube crane. - Xinjiang Agricultural Sciences 51(11): 19841989.

[37] Yang, Y., Liu, H., Qiu, D. (2017): Transcriptome data analysis of Stellera chamaejasme based on high-throughput sequencing. - Chinese herbal medicine 48(22): 4740-4747.

[38] Yuan, C., Fang, P., Zhong, W. J. (2017): Transcriptome sequencing and analysis of Phellodendron chinensis. - Chinese herbal medicine 48(21): 4507-4514.

[39] Zhang, D., Li, W., Li, D. (2009): The relationship between isoflavone content in soybean leaves and relative expression of PAL gene. - Soybean Science 28(4): 670-673.

[40] Zhang, Y., Zhou, Y., Chen, Q. (2014): Molecular basis of flowering time regulation in Arabidopsis. - China. Bull. Bot. 49: 469-482.

[41] Zhang, S. P., Zhang, S. H., Qiu, S. L. (2018): Based on the transcriptional sequence analysis of the related genes of sunflower green anthocyanin in purple back. - Acta Agriculturae Nucleatae Sinica 32(04): 639-645.

[42] Zhu, L. S., Liu, G., He, S. E. (2018): Analysis of the transcriptional characteristics of Eucalyptus grandis based on Illumina HiSeq 2000 sequencing technology. - Molecular Plant Breeding 13: 1-13. 